CANCER

\section{Small-molecule FGF trap shrinks tumours}

\begin{abstract}
The fibroblast growth factor (FGF)-FGF receptor (FGFR) system affects tumour growth, angiogenesis and drug resistance, and it is often aberrantly activated in cancer cells. However, the development of drugs that target the FGF-FGFR system has proved to be difficult. The FGF and FGFR family members are highly redundant and have pleiotropic effects, and blockade of FGFR signalling with tyrosine kinase inhibitors
\end{abstract}

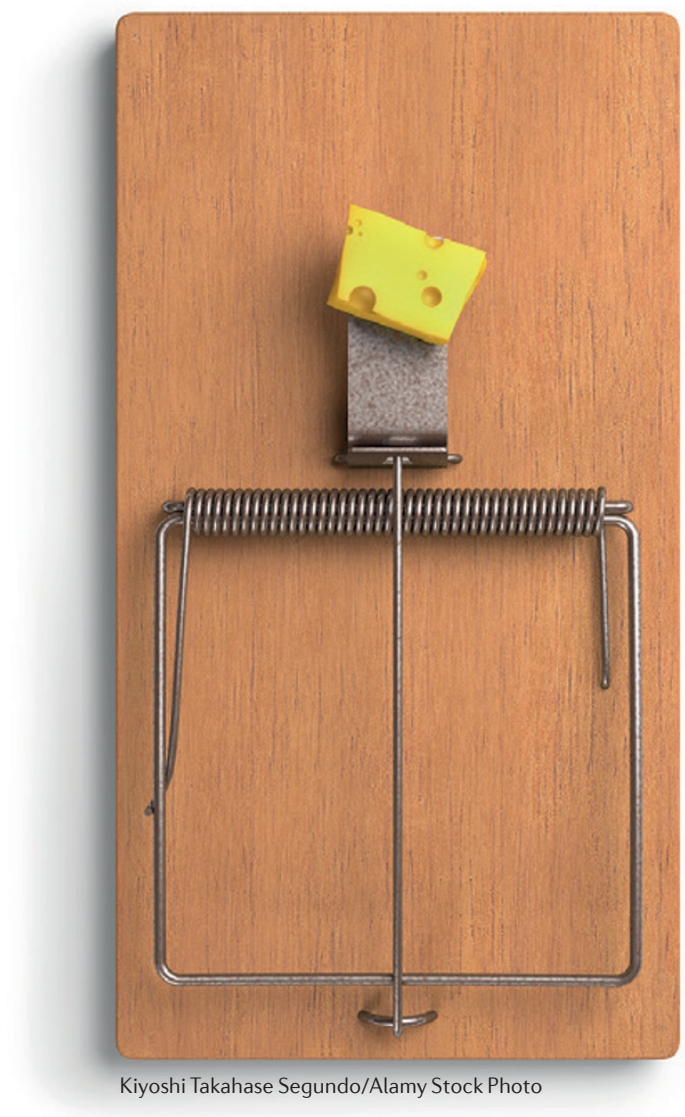

has been associated with toxicity. Now, reporting in Cancer Cell, Ronca and colleagues present a small molecule that acts as a multi-FGF 'trap', with anticancer activity and no signs of toxicity in animal models.

The authors first investigated the soluble pattern-recognition receptor long pentraxin 3 (PTX3), which binds various FGF family members and acts as a natural FGF trap. To test whether PTX3 has anticancer properties, transgenic $\mathrm{TgN}$ (Tie2hPTX3) mice were generated, which express human PTX3 (hPTX3) under the control of Tie2 (an endothelial cell-specific transcription regulatory sequence). In vitro experiments showed that endothelial cells from these mice have lower levels of FGFR1 phosphorylation upon stimulation with FGF2, indicating an inhibitory role of hPTX3.

Next, the mice were used in heterotopic and orthotopic tumour models. FGF-dependent melanoma, prostate, lung, pancreatic and mammary carcinoma cells had impaired growth rates and induced less neovascularization in $\mathrm{TgN}$ (Tie2-hPTX3) mice when compared to wild-type animals. FGF-dependent sarcoma and melanoma cells also showed a reduced metastatic potential (for the liver and lungs, respectively) of TgN(Tie2-hPTX3) mice. Conversely, homozygous inactivation of $P t x 3$ in mice led to enhanced FGF-dependent angiogenesis, tumour growth and metastatic spreading in tumour models.
In order to identify small molecules that may mimic the activity of PTX3, the authors determined a PTX3 minimal sequence - the acetylated pentapeptide ARPCA that binds to FGF2 and antagonizes its binding to FGFR. Pharmacophore modelling of the interaction of ARPCA with FGF2 was then used for in silico screening of a National Cancer Institute small-molecule database, leading to the identification of NSC12. This compound was found to bind to all members of the canonical FGF subfamilies, interfering with their binding to the four FGFR family members. Accordingly, NSC12 inhibited the proliferation of different FGF-dependent tumour cell lines, but had no inhibitory effect on FGFindependent tumour cells expressing a constitutively active FGFR1.

In vivo, NSC12, administered by intraperitoneal injection or oral gavage, was shown to inhibit FGFR phosphorylation, angiogenesis and primary and metastatic tumour growth of FGF-dependent human and mouse cancer cells. Importantly, there was no systemic toxic effect and, in contrast to the smallmolecule FGFR inhibitors that have been developed so far, there was no sign of hyperphosphataemia.

These data indicate that NSC12 is an interesting lead compound for the development of orally active smallmolecule FGF-trap therapeutics. Also, the authors point out that their data emphasize the possibility of exploiting the protein interactome for the design of orally active small-molecule multi-ligand traps.

Alexandra Flemming

ORIGINAL RESEARCH PAPER Ronca, R. et al. Long-pentraxin 3 derivative as a small-molecule FGF trap for cancer therapy. Cancer Cell 28, 225-239 (2015) 